\title{
Volume Changes in Protein Evolution
}

\author{
Mark Gerstein $^{1} \dagger$, Erik L. L. Sonnhammer ${ }^{1}$ and Cyrus Chothia ${ }^{1,2}$
}

\author{
${ }^{1}$ MRC Laboratory of Molecular Biology and \\ ${ }^{2}$ Cambridge Centre for Protein Engineering \\ Hills Road, Cambridge CB2 $2 Q H, U . K$.
}

\begin{abstract}
We have determined the variations in volume that occur during evolution in the buried core of three different families of proteins. The variation of the whole core is very small $(\sim 2.5 \%)$ compared to the variation at individual sites $(\sim 13 \%)$. However, by comparing our results to those expected from random sequences with no correlations between siles, we show that the small variation observed may simply be a manifestation of the statistical "law of large numbers" and not reflect any compensating changes in. or global constraints upon. protein secuences.

We have also analysed in detail the rolume variations at individual sites, both in the core and on the surface, and compared these variations with those expected from random sequences. Individual sites on the surface have nearly the same variation as random sequences $(24 \%$ versus $28 \%$ variation $)$. However. individual sites in the core have about half the variation of random sequences $\left(13 \%_{0}\right.$ versus: $30 \%$ o $)$. Roughly, half of these core sites strongly conserve their volume $(0$ to $10 \%$ variation): one quarter have moderate variation (10) to $20 \%$ ); and the remaining quarter vary randomly ( 20 to $40 \%$ ).

Our results have clear implications for the relationship between protein sequence and structure. For our analysis, we have developed a new and simple method for weighting protein sequences to correct for unequal representation, which we describe in an Appendix.
\end{abstract}

Keywords: random sequences: globins; dihydrofolate reductase:

plastocyanin-azurin family: tree weighting

\section{Introduction}

The determination of the atomic structures of myoglobin and haemoglohin showed that very different protein sequences could produce similar three-dimensional st ructures. This phenomenon was explained by the hypothesis that mutations in the interior are complementary. That is, the atoms added to. or lost from. the protein core because of one mutation are compensated by a subsequent mutation in the opposite direction (Kendrew \& Watson, 1966). In 1970 Lim \& Ptitsyn (1970) (arried out a calculation on the small number of globin sequences available at the time $(52)$ and showed that the volume of the 31 core residues was essentially constant. At the time this result was taken to support the complementary mutation hypothesis.

Later. Lesk \& ('hothia (1980) analysed the threedimensional structures of nine different globins. which had substantial differences in sequence. (The

† Present address: Beckman Laboratories for Structural Biology. Department of Cell Biology. itanford Medical School. Stanford. CA 94305 U.S.A. Internet: mbg@cb-iris.stanford.edu. minimum sequence identity between pairs was $16 \%$.) In the different globins the mean size of residues at homologous helix-helix interfaces varied in size by up to $50 \%$. This variation implied that the mutations between sequences were not locally complementary at a given interface. 'I'he proteins adapted to these changes by relative shifts in the position and orientation of the helices (up to $7 \AA$ and $20^{\circ}$ ) in a manner that conserved the structure of the haem pocket.

The analysis of other families of proteins demonstrated that, in general, proteins adapt to mutations by structural changes (Chothia \& Lesk, 1987), and the same view has emerged from protein engineering studies (Eriksson et al., 1992). However, it was not apparent how this view of protein evolution could be reconciled with the apparent "constancy" of the total volume of protein interiors found by $\mathrm{Lim} \&$ Ptitsyn.

Ptitsyn \& Volkenstein (1986) addressed this problem; by determining the volume variations at individual buried sites and for total core in the nine globin structures, they found that the core volumes of artificially generated random sequences were very close to those of observed ones. Thus, they showed that the apparent "constancy" in core volume over 
Table 1

Key sequences and their associated structures

\begin{tabular}{|c|c|c|c|c|c|}
\hline $\begin{array}{l}\text { PDB } \\
\text { identifier }\end{array}$ & $\begin{array}{l}\text { Key sequence } \\
\text { (SwissProt or } \\
\text { PIR identifier) }\end{array}$ & & Structure Reference & $\begin{array}{c}\text { Sequencet } \\
\text { identity } \\
\left({ }^{0} 0\right)\end{array}$ & $\|\left(*(*)_{+}^{+}\right.$ \\
\hline \multicolumn{6}{|l|}{ Globins } \\
\hline 2hhb & HAHI & Human Haemoglobin $x$-chain & Fermi of al. (198t) & $l(n)$ & $(1.10$ \\
\hline $2 \mathrm{hhb}$ & $\mathrm{HBHC}$ & Human Haemoglobin $\beta$-chain & Fermi ef al. (1984) & 45 & $(1.16)$ \\
\hline $2 \mathrm{lhb}$ & GGLMS & Sea Lampry Haemoglobin & Honzatko el al. (19855) & 37 & $11 \cdot 81$ \\
\hline $1 \mathrm{mbd}$ & MYWHP & Sperm Whale Myoglobin & Phillips \& Schoenborn (1981) & 28 & $(1)+11$ \\
\hline $2 \mathrm{hbg}$ & GGNWIB & Bloodworm Haenoglobin & Arents \& Love (1989) & 24 & 4.35 \\
\hline $1 \mathrm{mba}$ & GGGAA & Sea Hare Myoglobin & Bolognesi al al. (1989) & 18 & $\because 18$ \\
\hline lecd & GGICE3 & ('hironomous Haemoglobin & Steigemann \& Weber $(1981)$ & 18 & $1 \cdot 10$ \\
\hline $2 \ln 4$ & GPYL2 & Yellow Lupine Leghremoglobin & Arutyunyan ol al. (1980) & 18 & $1 \cdot(1) i$ \\
\hline \multicolumn{6}{|c|}{ Plastocyanin-azurin family (Plas.-Az.) } \\
\hline $2 p c y$ & ('UPX & Lombardy Poplar Plasteryanin & (iuss \& Freeman (1983) & I(x) & 1.03 \\
\hline $2 a z a$ & AZALCD & A. denitrificans Azurin & Baker (1988) & 2.2 & $1 \cdot 01$ \\
\hline \multicolumn{6}{|c|}{ Dihydrofolate reductases (DHFR) } \\
\hline ldhl & DYR HLMAN & Human DHFR & Davies of al. $(1993 a)$ & $100)$ & $0 \cdot 131$ \\
\hline $8 d f r$ & DYR CHICK & ('hicken DHFR & Davies of al $(1993 b)$ & 79 & $(1.8)$ \\
\hline $4 \mathrm{dfr}$ & DYRĀ ECOLI & E. coli DHFR & Bolin al al. (1982) & 35 & 1.08 \\
\hline $3 \mathrm{dfr}$ & DYR $\bar{L} A C C A$ & L. rasei DHFR & Bolin et al. (198:) & 20 & $1-33$ \\
\hline
\end{tabular}

† Percentage sequence identity to first key sequence in family (e.g. HAH(').

\$ The weight $w(s)$ assigned to this particular sequence in the context of all the secquences in the family. The average weight of eath sequence is 1.0 .

evolution may result from general statistical properties of sums of random numbers.

Here we greatly extend Ptitsyn \& Volkenstein's work; we have determined the volume variations at the individual buried sites and for whole buried core in 568 globin sequences. We have also carried out the same calculations on two other protein families, the dihydrofolate reductases and the plastocyaninazurin family. The three protein families have very different core sizes and structures: the plastocyaninazurins (also known as the cupredoxins, see Adman, 1991) are all $\beta$-proteins with small cores $\left(\sim 4000 \AA^{3}\right)$; the globins are all- $\alpha$ proteins with medium-sized cores $\left(\sim 6000 \AA^{3}\right)$; and the dihydrofolate reductases are $\alpha / \beta$ proteins with large cores $\left(\sim 8000 \AA^{3}\right)$.

Our calculations show that the particular results obtained by Ptitsyn \& Volkenstein on a small number of globin structures are generally true for protein families with widely divergent sequences. Namely, we show that while individual sites in the protein core vary appreciably in volume (some by up to $40 \%$ and on average by $\sim 13 \%$ ), the volume of the whole core is nearly constant (varying $\sim 2.5 \%$ ). Moreover, we show that uncorrelated mutations at the individual core sites can reproduce the observed variation in core volume, so it is not necessary for mutations to be locally compensating to produce the small observed variation in core volume.

We do two further sets of calculations; we determine the volume variations at surface sites and those that would be produced by random changes in sequence. Comparison of the different calculations shows the variation at individual sites on the surface is nearly what is expected for random sequences. At individual buried sites, however. the average variation is less than half of what is expected for random sequences. This disparity is due to the local size constraints imposed by the protein structure on particular buried sites. These constraints vary greatly: some sites are absolutely conserved, whilst others essentially vary like random sequences.

\section{Methods}

\section{(a) Protein segueness and their aligmment}

Protein sequences were taken from swissl'rot-24 (Bairoch \& Boeckmann. 1992) and PIR-36 (Barker ot al.. 1992). and protein structures were taken from the Protein Data Bank (Bernstein of al. 1977). In total we collected 24 plastocyanin-azurin sequences. 568 globin sequencess and 4 () dihydrofolate reductase sequencess.

The sequences for the three families were alignedt based on "key" sequences of known structure. Bashford ot al. (1987) described the application of this alignment procedure to 226 globin sequences. The key sequences corresponding to 8 known globin structures were first aligned based on structural superimposition. and then the rest of the sequences were aligned to the keys. As shown in Table 1 , for our work the globin alignment in Bashford et al. (1987) was expanded to include more sequences. New alignments were constructed for the dihydrofolate-reductase and plasteryanin-azurin families based on the key. sequences corresponding to known structures.

The buried and surface sites in the 3 families are listed in Table 2. With one or two exceptions discussed in the

† The sequence alignments and computer programs used for this work will be made available electronically by email to mbg@ucb-iris.stanford.edu or by anonymous ftp to cb-iris stanford edu or cele mrc-lmb.cam.ac.uk. 
Table 2

Residue sites constituting the core and the surface

\begin{abstract}
Gilobin core

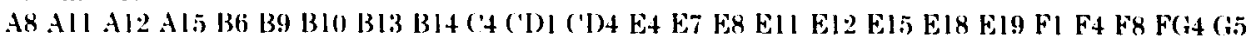

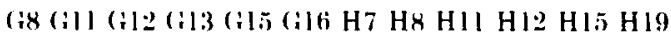

Gilobin surface

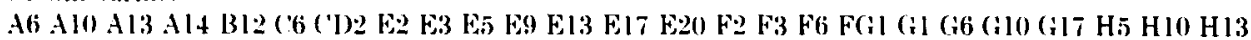
$\mathrm{H} 9$

Dihydrofolate reductase core

$5-11,15-17,24,27,30,33,34,38,49-53,56-57,60,67,70,83,86,90,93,96,100,112-117,120,121,124$, 1333-136. 138. 148. 156. 177, 179, 181. 182.

Plastocyanin-azurin core

1. 3, 5. 14, 19, 21, 27, 29, 31, 33, 37-41, 72, 74, 80, 82, 86, 86, 92, 94, 96.

List of the residues forming the core in each of the three families studied and forming the surface in the globins. In the structures used for the alignment. i.e. those corresponding to the key sequences. residues in the core had. on average. less than $15 \AA^{2}$ of accessible surface. In addition. we included sites that had higher mean accessible surface areas, between $15 \mathrm{~A}^{2}$ and $20 \mathrm{~A}^{2}$. and whose side chains pointed into the protein interior. Surface residues had at least $50 \mathrm{~A}^{2}$ of accessible surface. For the globins, this definition closely paralleled that in Bashford et al. (1987).

For the globins. residues are numbered according to the canonical numbering scheme introduced by Kendrew (Fermi \& Perutz, 1981) and described in Bashford ef al. (1987): for the dihydrofolate reductases, residue numbering refers to the human sequence (DYR IIL'MAN); and for the plastoryanin-azurin family. numbering refers to the poplar plastocyanin sequence (('LPX).
\end{abstract}

table raption. core residues were defined as those that ocrupied sites whose mean accessible surface area (Lee \& Richards. 1971) in the key structures was less than $10 \AA^{2}$. Various other accessibility cutoffs were tried but not found to make appreciable difference to the results. Siufare sites were defined as those with more than $50 \mathrm{~d}^{2}$ of accessible surface.

For the structures. volumes were calculated according to the Richards implementation of the Foronoi method (Richards. 1974). For the sequences. standard volumes for each residue type were taken from Harpaz et al. (unpublished results) and are reproduced in Table 3.

\section{(b) A treighting wheme for the comparison of sequence feralures}

As described in Table 4 , for a given site. we a veraged the standard residue volumes over all sequences in each alignment to get a mean volume for that site and variation about this mean. Throughout the text. we express this variation as a percentage standard deviation (percentage S.I).t). Likewise. for each sequence we summed the standard residue volumes of the buried sites to get a core volume, and then we averaged these core volumes over all the sequences to determine a mean core volume and variations about this mean.

To compensate for the unequal representation of the sequences in our alignment, we used a weighting scheme that gave low weights to closely related sequences. such as the haemoglobin $\alpha$-chains in the globin alignment. This weighting scheme is deseribed in the Appendix. It gave us greater confidence in the quantitative accuracy of our conclusions. However. it did not change our conclusions or results significantly as compared to those reached from doing unweighted averages.

† Abbreviations used: S.D.. standard deviation: DHFR, dihydrofolate reductases: Plas.-Az. Plastoeyanin-Azurin family: C'PL', central processor unit.
Table 3

standard residue volumes and frequencies

\begin{tabular}{|c|c|c|c|}
\hline \multirow[b]{2}{*}{$\begin{array}{l}\text { Residue } \\
\text { type }\end{array}$} & \multicolumn{2}{|c|}{ Frequency } & \multirow[b]{2}{*}{$\begin{array}{c}\text { Standard volumes } \\
\qquad V_{1}\left(A^{3}\right)\end{array}$} \\
\hline & $\begin{array}{c}\text { This workt } \\
f_{\text {tr }}\left({ }^{\circ}{ }_{0}\right)\end{array}$ & $\begin{array}{c}\text { Janin }(1979) \ddagger \\
\int_{1 r}\left({ }^{\circ}\right)\end{array}$ & \\
\hline (ily & $11 \cdot 1$ & 11.8 & 64 \\
\hline $\mathrm{Ala}$ & 13.4 & $11 \cdot 2$ & 90 \\
\hline Val & $13 \cdot 4$ & $12 \cdot 9$ & 139 \\
\hline Leu & $12 \cdot 6$ & $11 \cdot 7$ & 164 \\
\hline Ile & $8 \cdot 8$ & 86 & 164 \\
\hline Pro & $2 \cdot 6$ & 2.7 & 124 \\
\hline Met & $2 \cdot 5$ & 1.9 & 167 \\
\hline Phe & $6 \cdot 2$ & $5 \cdot 1$ & 193 \\
\hline$T_{Y r}$ & $3 \cdot 1$ & $2 \cdot 6$ & 197 \\
\hline Trp & 1.9 & $2 \cdot 2$ & 231 \\
\hline Ser & 58 & 80 & 95 \\
\hline Thr & 5.5 & $4 \cdot 9$ & 121 \\
\hline Asn & 1.5 & $2 \cdot 9$ & 126 \\
\hline Cys & $4 \cdot 1)$ & $1 \cdot 6$ & 113 \\
\hline His & $1 \cdot 6$ & $4 \cdot 1$ & 159 \\
\hline (ilu & $1 \cdot 4$ & 20 & 142 \\
\hline Asp & $3 \cdot 0$ & 1.8 & 118 \\
\hline Arg & 0.9 & $2 \cdot 9$ & 195 \\
\hline Lys & 0.7 & 0.5 & 170 \\
\hline
\end{tabular}

† Frequency of 20 residue types in buried residues in proteins. The frequencies were defined by counting the number of buried residues in a database of 119 protein crystal structures. Structures in the database all were solved to very high resolution (between 1.0 and $1.9 \mathrm{~A}$ ), had $R$-factors below $20 \%$, and had good stereochemistry as defined by Morris et al. (1992). Buried residues were defined as those with less than $15 \mathrm{~A}^{2}$ accessible surface area. This column of numbers was used for frequency distribution (2) in Table 8A.

† Our residue frequencies shown in the first column are very similar to those in Janin (1979), an earlier determination of the frequencies of buried residues. which was based on the fewer high resolution structures known at the time.

$\$$ Standard Voronoi volume for each residue type, taken from Harpaz, Gerstein \& Chothia (unpublished results). 


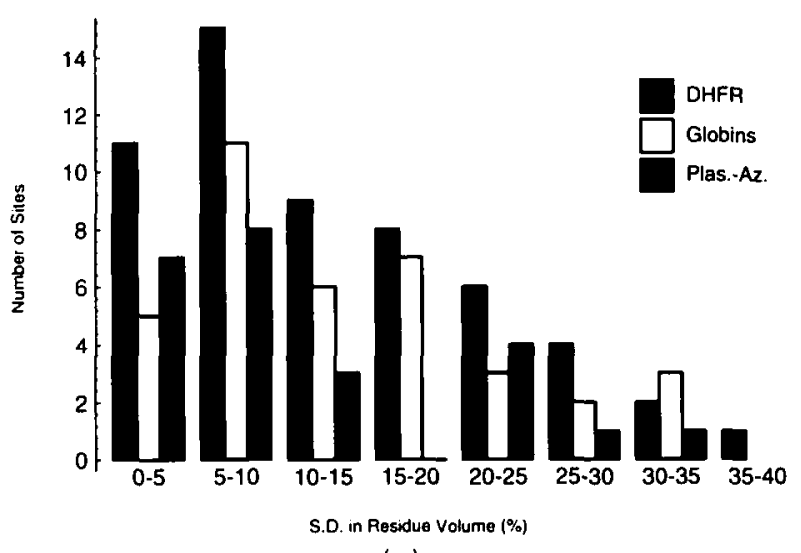

(a)

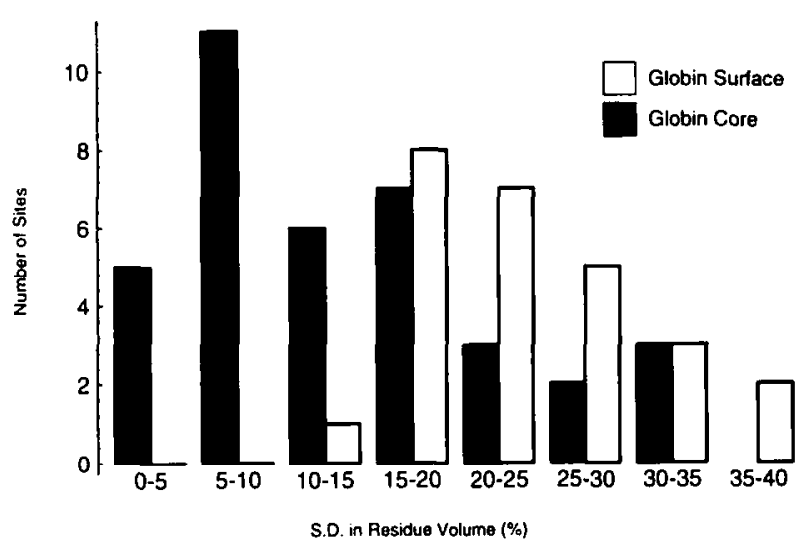

(b)

Figure 1. Volume variation at individual sites. (a) Histogram of the variation in volume of the individual ('ore sites $\left(\sigma_{r} / V_{r}\right)$ in each of the 3 families (filled bars. dihydrofolate reductases: open bars, globins: grey bars. plastocyanin-azurin family). Note the similarity between the families. The mean volume variation at individual sites $\left\langle\sigma_{r} / \Gamma_{r}\right\rangle$ is $14 \%$ for the globins. $11 \%$ for the plastoryanin-azurin family, and $13 \%$ for the dihydrofolate reductases. (b) Histogram comparing the volume variation of individual globin sites $\left(\sigma_{r} / V_{r}\right)$ in the core (filled bars) and with those on the surface (open bars). The major difference is that there are no sites with small $\left(\sim 10^{\circ}\right)$ volume variation on the surface.

of structures is less than $2.6 \%$. Figure 2 shows that this apparent constancy in core volume covers the whole range of sequence identities.

Table 6 also shows that while the three protein families have different numbers of residues in the core, the average size of a core residue in all three families is essentially the same: $\sim 150 \AA^{3}$ or seven to eight non-hydrogen atoms (i.e. between $\mathrm{Val}$ and Leu in size).

The constancy of core volume discussed in the preceding paragraphs is derived from applying the standard residue volumes to protein sequences. We also carried out the calculation in the reverse direction and directly determined the core volume for eight globin structures (Table 7). Corroborating our sequence calculations, the structure calculations have similar volume variations.
Table 6

Observed volume variation of the whole core

\begin{tabular}{|c|c|c|c|}
\hline & Globins & Plas. -Az. & DHFR \\
\hline Number of buried residue sites, $R$ & 37 & 24 & 56 \\
\hline Number of sequences. $N$ & 568 & 40 & 24 \\
\hline Average core volume, $r\left(A^{3}\right)$ & 5607 & 3616 & 8201 \\
\hline$S . D$. in core volume, $\frac{\sigma}{V}\left({ }^{\circ}\right)$ & $2 \cdot 6$ & $1 \cdot \overline{5}$ & $2 \cdot 2$ \\
\hline $\begin{array}{l}\text { Average core volume per } \\
\text { residue }\left(A^{3}\right)\end{array}$ & 152 & 151 & 146 \\
\hline
\end{tabular}

The total volume of the whole core varies less than $2.6{ }^{0}$ in each of the 3 families studied. Moreover, the average core residue in each of these 3 families is roughly similar in volume. It contains 7 to 8 atoms and is roughly between $V a l$ and Leu in size.

\section{Volume Variation Produced by Random Sequences}

In the preceding section we found that, for each of the three families, the large volume variations at individual core sites ( 11 to $14 \%$, on average) tend to cancel to give a small variation for the total core volume $(1.5$ to $2 \cdot 6 \%)$. To put our results in context, we calculated the volume variations that would be produced by random sequence changes that have no correlations between sites.

For these calculations, we supposed that all the sites in the core were filled with residues picked at random from identical distributions of amino acids. In this case, all the sites are uncorrelated and equivalent, so the variation in the volume of the whole core (measured as a percentage S.D.) is just $1 / \sqrt{R}$ of the variation at a single site, where $R$ is the number of residue sites. Clearly, the crucial parameter is the variation at a single site, which, in turn, depends on the residue frequencies used in the generation of random sequences. As shown in Table 8, we tried three different types of frequency distributions:

(1) A uniform distribution, where all residues have equal frequency. This is the simplest scheme and introduces no a priori constraints. The volumes of random globin sequences constructed according to this distribution are shown in Figure 3. This distribution is somewhat unrealistic because it allows charged residues into the core.

(2) General distributions that take into account the chemical character of buried residues. The simplest such distribution is another uniform distribution, which just excludes the residues that are rarely buried: charged residues and GIn and Asn. A more accurate distribution is shown in Table 3 . It is derived from the frequencies of buried residues in 119 protein crystal structures. The major problem with these general buried residue distributions is that the residue frequencies found in particular proteins may be influenced by their secondary structures and topologies.

(3) Distributions based on the specific buried residues in each of the three protein families. These were made by compiling frequency tables similar to the one shown in Table 3 for the buried residues in each protein family. These one-family distributions 


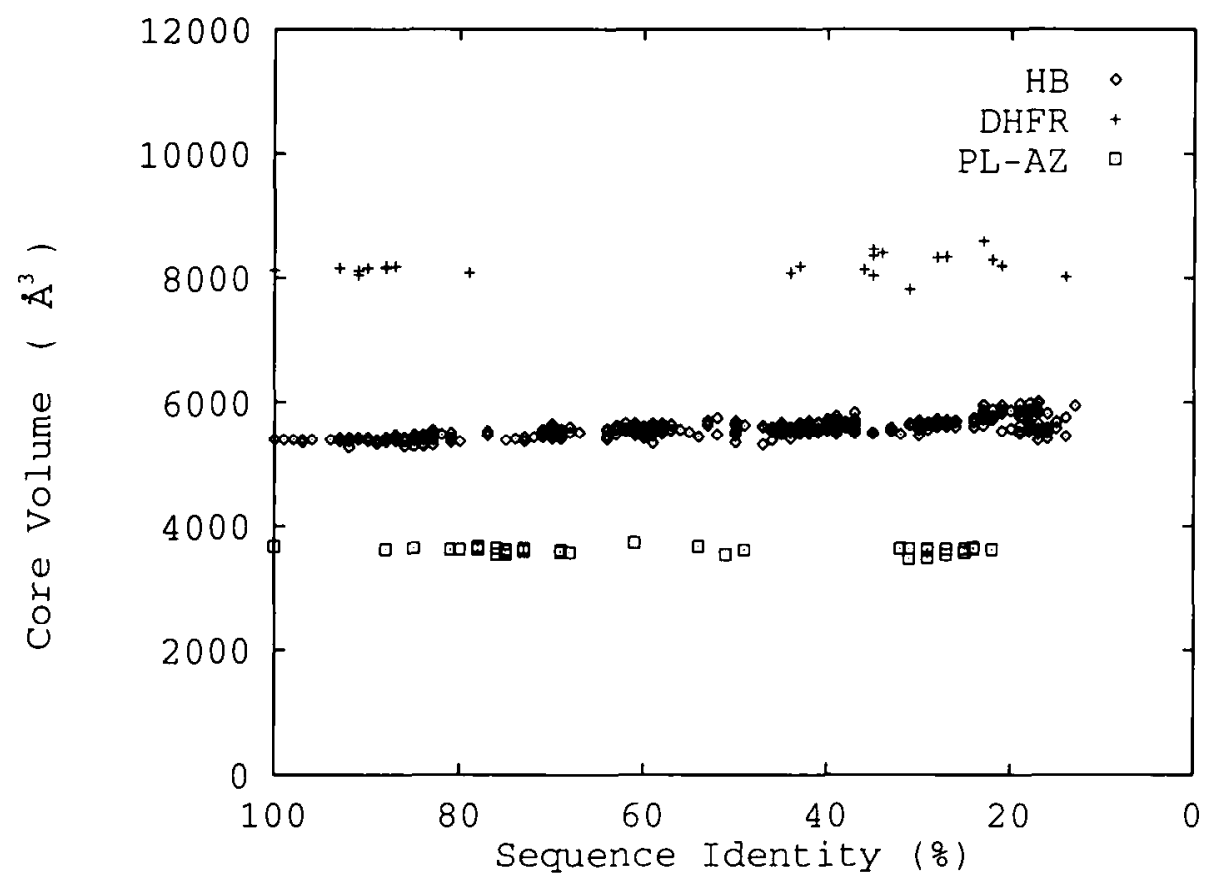

Figure 2. Core volume variation versus sequence identity. The volume of the core rersus sequence identity to the first key sequence in each of the 3 protein families. The smallest core belongs to the plastocyanin-azurin family. followed by the globin family, and then the dihydrofolate reductase family. For these 3 families. secuence identity was computed relative to the key sequences: AZALCD. HAHC and DYR_HLMAN, respectively (see Table 1). For purposes of comparison, the volume of a methyl group is $26 \AA^{3}$.

obviously do not suffer from the same problems as the other two types of distributions. However, they introduce a significant amount of a priori bias into the generation of any random sequence.
Despite the differences between the three types of frequeney distributions. they essentially give the same result: the calculated rariations in site size (24 to $31 \%$ ) are roughly double the average observed

Table 7

('omparison of sequence and structure calculations for the globins

\begin{tabular}{|c|c|c|c|c|c|}
\hline $\begin{array}{l}\text { Sequence } \\
\text { identifiert }\end{array}$ & $\begin{array}{l}\text { Sequence } \\
\text { volume. } I^{\prime} \text {, } \\
\left(A^{3}\right) t\end{array}$ & $\begin{array}{l}\text { Structure } \\
\text { identifiert }\end{array}$ & $\begin{array}{l}\text { Structure } \\
\text { volume. }{ }^{\prime} \\
\left(A^{3}\right) \S\end{array}$ & $\begin{array}{l}\text { Differencer } \\
\text { from mean } \\
\text { for sequences } \\
\left.\left(V_{3}-V^{\circ}\right) / V^{\prime}\right)\left(0_{0}\right)\end{array}$ & 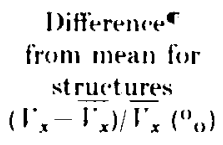 \\
\hline HAHL: & 5402 & $2 \mathrm{hhb}$ & 5941 & $-2 \cdot 9$ & $-1 \cdot 4$ \\
\hline HBHE & $55+4$ & thhb & 6001 & -0.4 & -0.4 \\
\hline GGLMS & 5518 & $2 \mathrm{lhb}$ & 5959 & $-(1 \cdot 9$ & $-1 \cdot 1$ \\
\hline MYWHP & 56660 & $1 \mathrm{mbd}$ & 6117 & 1.7 & $1 \cdot \tilde{5}$ \\
\hline (GCNWIB & 56000 & $2 \mathrm{hbg}$ & 6169 & $0 \cdot 6$ & 24 \\
\hline$G(\mathrm{MGAA}$ & 5990 & $\operatorname{lmb} a$ & $6+19$ & $7 \cdot 6$ & $\mathbf{b} \cdot \mathbf{i}$ \\
\hline GGICE3 & 5878 & led & $64+4$ & 566 & 7.0 \\
\hline (iPYL2 & 5608 & 2 lht & $61+5$ & 0.8 & $\geq 0$ \\
\hline
\end{tabular}

t Sequences and structures correspond to the key sequences used in the globin aligmment (Table 1).

$\$$ Volume $\left(\AA^{3}\right)$ of the core of a particular globin sequence. For each of the 37 buried sites (Tab)e 2 ). the standard residue volumes (Tuble 3 ) were used to calculate a volume.

$\$$ Volume $\left(A^{3}\right)$ of the core of a particular globin $X$-ray ervstal structure $\left(V_{x}\right)$. For each of the 37 residues that formed the core, the atoms in the core were used for Voronoi polyhedra culculations. Our implementation of Richards' (1974) Voronoi polyhedra programme was used for the calculations. In all cases the structure volumes will be more than the sequence volumes $\left(l_{s}\right)$ and are not directly comparable. This disparity exists because the core residue definition in Table 2 is based on the average of all the structures. Any individual structure will, therefore, always expose to solvent a few of the atoms thought to be in core, and this exposure will tend to enlarge the calculated Voronoi polyherlra and increase the calculated volume.

For the sequences, the percentage difference of a particular sequence volume $l_{s}$ compared to the mean core volume $V^{\prime}$ (defined in Table $t$ and shown in Table 6 ) is listed. For the structures, percent age difference in a particular structure volume $V_{x}$ compared to the mean value of ${ }^{*}$ for all 8 st ructures is listed. As they have been normalized to compensate for the smaller average size of the structure volumes, these two percentage differences are comparable. "They show that volume variation of the core calculated for the structures $\left(-2\right.$ to $\left.+7 \%_{0}\right)$ is comparable to that calculated for the seyuences $(-3)$ to $+8 \%)$. 
Table 8

('omparisom of the obsercert wariation with that of random sequencess

A. Core sile's

Observed values (from Table 6 and Fig. $1(a)$

Number of core sites. $R$

Mean S.I). of individual sites. $\left\langle\frac{\sigma_{r}}{r_{r}}\right\rangle\left({ }^{\prime}{ }_{0}\right)$

Globins Plas.Aa, DHFR

S.I). in core volume. $\frac{\sigma}{j}\left({ }^{\prime \prime}, 0\right)$

Random secquencess, assuming sites vary

\begin{tabular}{|c|c|c|}
\hline 37 & $\underline{4}$ & 56 \\
\hline 1.4 & 11 & 13 \\
\hline $2 \cdot 6$ & 1.5 & $2 \div 2$ \\
\hline 27 & 27 & 27 \\
\hline $4 \pi$ & 56 & $3 \cdot 7$ \\
\hline 31 & 31 & 31 \\
\hline (0) & 6.4 & +3 \\
\hline 24 & $\because 6$ & 30 \\
\hline $4: 2$ & 4.9 & +1 \\
\hline
\end{tabular}

independently and randomly according to the following frequency distributions (same $f_{t r}$ for all sites)†

1. V'niform distribution of all 20 amino acids S. 1). of individual sites $\left({ }^{\circ}, 1\right)$ Fixperted $s$. I). in rore rolume (" $\left.{ }^{\circ}\right)$

$\because$. Distribution of buried residues in an average protein

S.L. of individual sites ("a)

Experted si.1). in core volume $\left({ }^{\circ}{ }_{0}\right)$

3. I Distribution of hurjed resiclues in a particular protein family:

S.D. of individual sites (")

Expected S.D. in core volume (")

$+2$

$4 \cdot 9$

30
$+\cdot()$

13. Alobin surface sites

(libserved values

Number of surface sites

Mean S.L) of individual sites ("o)

s.1). in total volume of the sites $\left({ }^{\circ}{ }_{10}\right)$

Random sequences, assuming the surface sites

vary independently and randomly according to a uniform distribution of the 13 non-

hrodrophobic resiclues

S.D. of individual sites ( $\left.{ }^{\circ}\right)$

Expected s.I). in total rolume ("n)

A shows comparisons between the observed variane (or. rather. standard reviation) of the core and expected rariances based on different random sequences. The Fischer F-test can be used to test whether the difference hetween the two variances is significant. Application of this test to all the comparisons in this Table, using $Y-1$ degrees of freedom for the observed variances and $(R-1)(X-1)$ degrees of freedom for the expected variances. shows that the differences between the variances are all signiticant.

The calculations in $B$ are completely analogous to those in A but they are carried out on the globin surface sitex. (ienerating random surface secquences consists of picking a residue from the 13 nonhydrophohic amino acids ( $A$ A P Y S T N Q H E D R K), where each of these amino acids has an equal whance of being picked. ('learly there is much better agreement between the observed and calculated variation for surface sites than for buried sites.

$\dagger$ As all sites $r$ are equivalent for the random distributions. the expected percentage standard deviation of the whole core. $\sigma / l$, is directly related to the percentuge standard deviation of an individual site $\sigma_{r} / l$, by the equation:

$$
\frac{\sigma}{\mathrm{l}}=\frac{\mathrm{l}}{\sqrt{R}} \frac{\sigma_{r}}{l_{r}}
$$

The experted deviation and mean volume of a site. $\sigma_{r}$ and $l_{r}$, are, in turn. calculated according to residue frequencies $f_{i r}$ and standard volumes $V_{1}$, as shown in Table 4 . Depending on one s assumptions about the distribution of amino arids in the protein core. 3 different sets of residue frequencies can be used.

1. For a uniform distribution of amino acids, a residue is picked at random from 20 equi-probable amino acids, i.e. $f_{t r}=1 / 20$ for all $t$ and $r$.

2. For the distribution of buried residues in average protein, a residue is pirked from the 20 amino acids according to the frequency distribution for buried residues in 119 high-resolution crystal structures, i.e. $f_{t}$ is taken from the second column of Table 3 . This distribution gives nearly the same results as a uniform distribution of uncharged and neutral amino acids. where a residue is picked from one of the 14 equi-probable amino acids. ( 6 A Y L I P .II F Y W S T ( $\mathrm{H}$ ).

3. For the distribution of buried residues in a particular family. for each of the 3 protein families, the types of residues owurring in the core in all the sequences are counted and used to make a frequency distribution similar to the one shown in Table 3. Then a residue is picked according to these frequencies. That is, for each protein family (e.g. for the haemoglobins), the actual frequencies for residues of type $t$ to be at site $r$. $\int_{i r}^{*}$. are averaged over all sites to give $f_{t r}$ :

$$
f_{t r}=\frac{1}{R} \sum_{r=1}^{R} f_{t r}^{*}
$$

In this expression, the actual frequencies $f_{t r}^{*}$ are different at each site (as in Table 9), while the averaged frequencies $f_{t r}$ are the same at each site. The suhseript $r$ is kept in $f_{t r}$ to he consistent with the notation in Table $t$ and to emphasize that $f_{t r}$ refers to frequencies at an individual site. 


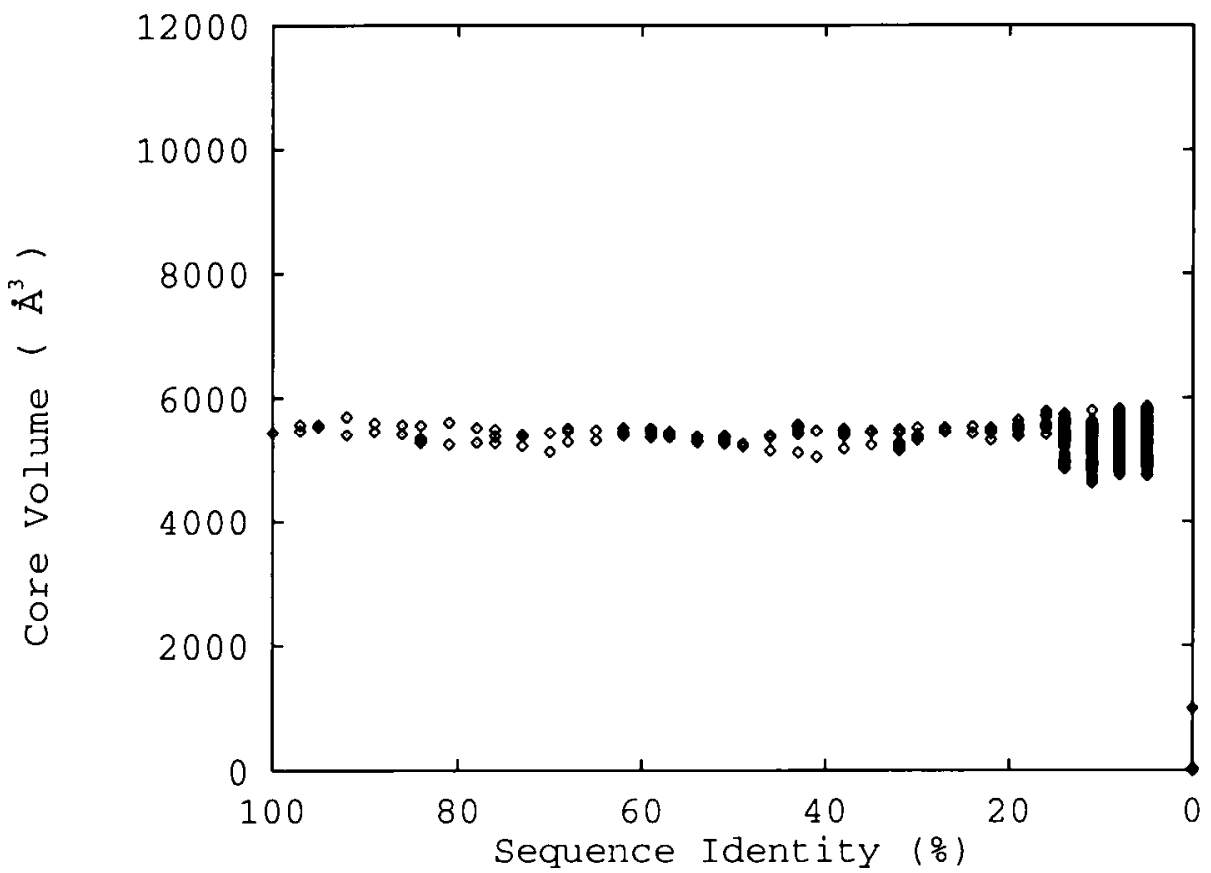

Figure 3. Volume variation of random sequences. The volume in the core rersus sequence identity to one particular sequence for a random sequence. The sequence contained 37 sites, the same number as the globin core. and was generated by picking residues from a uniform distribution of amino acids. i.e. where each residue type is equi-probable. The sequence has a mean volume very similar to that of the globins ( $144 \AA^{3}$ per residue) but has a standard deviation roughly twice as large.

variations in site size (11 to $14 \%$ ). The single-site variations imply that for random sequences the expected variation in core volume is 4.0 to $6.3 \%$. which is roughly double the observed variation $(1.5$ to $2 \cdot 6^{\%}$ ).

Although the simple random distributions discussed above do not reproduce the observed variation for the core sites, they do describe the behaviour at the surface sites fairly well. As shown in Table $8 \mathrm{~B}$. the observed volume variation for individual sites on the surface of the globins $(24 \%$ on average) is close to that calculated from filling these sites with randomly chosen. non-hydrophobic residues $(28 \%)$.

\section{The Relationship Between the Observed Single-site Variations and the Observed Core Variation}

In the preceding section we assumed that all sites varied independently and randomly, according to the same hypothetical frequency distribution. Here we take the observed variation at the individual sites and calculate the expected variation for the whole core. That is, we again assume that the sites vary independently and randomly, but this time each site varies differently according to a frequency distribution that is derived solely from the amino acid residues occurring at that site in the sequence alignment. The variations in the volume of individual sites would in this case be the same as the observed values shown in Figure l(a).

Table 9 shows the result of our new set of assumptions; the calculated variation in core volume is similar to that observed. For the globins and the dihydrofolate reductases. the calculated and observed values were very close: $2.4^{\circ}$ o rersus $2.60^{\circ}$, for the globins and 2.00 o versus $2 \cdot 2 \%$, for the di-

Table 9

Relationship of the observed single-site cariutions to the observed variation of the whole core

(ilobins Plas.-Az. DHFR

\begin{tabular}{|c|c|c|c|}
\hline \multicolumn{4}{|l|}{$\begin{array}{l}\text { (Observed values (from Table } 6 \\
\text { and Fig. } 1(a) \text { ) }\end{array}$} \\
\hline $\begin{array}{l}\text { Number of Sites, } R \\
\text { Mean S.D. of individual }\end{array}$ & 37 & 24 & 56 \\
\hline sites. $\left\langle\frac{\sigma_{r}}{J_{r}}\right\rangle\left(\sigma_{0}\right)$ & 14 & 11 & 13 \\
\hline S.I). in core volume, $\frac{\sigma}{\Gamma}\left({ }^{0}{ }_{0}\right)$ & $2 \cdot 6$ & $1 \cdot 5$ & 20 \\
\hline $\begin{array}{l}\text { Assuming sites vary } \\
\text { independently and } \\
\text { randomly according to the } \\
\text { (different) observed } \\
\text { frequency distributions for } \\
\text { each site (different } f_{\text {or }} \text { for } \\
\text { each site) } \\
\text { Expected S.D. in core }\end{array}$ & & & \\
\hline volume. $\frac{\sigma}{\mathrm{l}}\left({ }^{\circ} \mathrm{o}\right) \dagger$ & $2 \cdot 4$ & $2 \cdot 5$ & $2 \cdot 0$ \\
\hline
\end{tabular}

t The variances of uncorrelated distributions add, so the expected percentage standard deviation of the whole core if the sites varied independently would be:

$$
\frac{\sigma}{V}=\frac{1}{V} \sqrt{\sum_{r=1}^{R} \sigma_{r}^{2}}
$$


hydrofolate reductases. For the plastocyanin-azurin family the calculated and observed values $(2-5)$ versus $15 \%$ ) are not as close. This discrepancy may reflect the bimodal nature of the family: the different plastocyanin sequences have considerable similarity (i.e. sequence identity) to each other, as have the azurin sequences, but between the two groups the similarity is low.

Thus, provided the residues are picked acroording to the correct distribution. filling the sites randomly gives nearly the same variation in core volume as that observed over evolution. It is not necessary to invoke compensating changes and correlations between sites to explain the apparent constancy in total core volumes. It is simply a statistical effect of the law of large numbers, i.e. in averaging random numbers, the average variation decreases as the sample size increases.

Since the average size of a buried residue in all three protein families (Table 6) is the same, our conclusion that the observed variation in core volume can be reproduced by random variations implies that the overall volume of the core is not strongly dependent on the particular sequence of the residues at the buried sites. The size of core mainly depends on the number of resiclues that point inward from solvent.

\section{Conclusion}

We have quantitatively determined the volume variations that occur in the buried cores of three protein families. Although the families have quite different structures and functions. the three families give very similar results. This suggests our results generally represent what occurs in protein families where sequence has diverged but function has been retained.

The variation in the total volume of the buried core is very small $\left(1.5\right.$ to $\left.2 \cdot 6^{\circ}\right)$ compared with the variation at individual sites (11 to $14^{\circ}$ o on average). However. we come to a conclusion similar to that of Ptitsyn \& Volkenstein (1986). that this apparent constancy of core volume does not necessarily. reflect any compensating changes in, or global constraints upon, protein sequences that have evolved from a common ancestor. Rather, it is consistent with appropriately chosen random sequence changes and as such is simply a manifestation of the statistical law of large numbers.

We compared the volume variations observed at individual sites with those produced by the random sequences generated according to standard frequency distributions. The observed variation at individual surface sites is similar to that produced by random sequences. In contrast. the observed variation at individual core sites $\left(\sim 13^{\circ} \%\right.$ on average) is roughly half that found for the random sequences $(\sim 27 \%)$.

The smaller variation found for core sites arises from their being subject to steric constraint in differing degrees (Fig. l(b)). About half the core sites conserve their volumes ( $<10^{\circ}$ o variation); about a quarter have moderate volume variation (10 to $20^{\circ}$ ); and the remaining quarter vary as much as random sequences ( 20 to $40 \%$ ).

Proteins in the same family have a common core whose structure is shared by all family members and peripheral regions whose structures vary between different members. For the three families discussed here, roughly one third of the residues in the "common core" are buried ('hothia \& Lesk, 1986). Thus, as we found (above) that half of the buried residues have strong volume constraints, approximately one sixth of the residues in the common core are greatly constrained in volume.

Our results have implications that highlight both the random and the invariant features of protein sequences:

\section{(a) Random features of protein sequences}

On one hand, our conclusions imply that because of general statistical considerations, a given core rolume depends mainly on the number of, and not on the type of. residues in the core. Thus, allowing for the specific size constraints on a few individual sites, residues drawn from a wide range of sequences would be acceptable for a particular core structure. These implications support the view that protein sequences are random heteropolymers, which are "edited" only slightly by evolution (Ptitsyn \& Volkenstein, 1986), and that the structural features of known protein folds can accommodate a wide range of sequences (Finkelstein \& Ptitsyn, 1987; Murzin \& Finkelstein. 1988: Finkelstein et al., 1993).

\section{(b) Sequence determinants of protein folds}

On the other hand, our conclusions do not imply that there are no volume constraints upon protein sequences in the core. We have found that some individual core sites are nearly invariant in volume and others have only small volume variations.

Furthermore, we have presented a procedure that gives a precise, quantitative description of this volume conservation at each site in families of sequences. The aspects of sequences that determine structure (and function) should appear as conserved features in families of related sequences. Our procedure can easily be adapted to quantify other conserved features, such as hydrophobicity and charge. Thus, it will allow one to derive a detailed picture of the conserved features in families of sequences. Examination of these features, and of their structural and functional role, should greatly extend our understanding of the relation between sequence and three-dimensional structure.

We thank R. Durbin and G. Mitchison for many helpful suggestions, particularly on statisties and the weighting scheme. We thank A. Lesk, Y. Harpaz, S. Brenner, S. Barrie, and $J$. Baldwin for reading the manuseript. M. G. acknowledges support from a Herchel-Smith Fellowship. 


\section{APPENDIX}

\section{A Method to Weight Protein Sequences to Correct for Unequal Representation}

To avoid misleading results due to the unequal representation of sequences in a multiple sequence alignment (Felsenstein, 1985), it is desirable to reduce the weight of over-represented sequences (Altschul et al., 1989; Sibbald \& Argos, 1990). The idea of most weighting schemes is that sequences located in densely populated regions of sequence space should get a lower weight than sequences in sparsely populated regions (assuming that the theoretical "true" distribution is flat and has a centroid that coincides with the centroid of the observed distribution). For instance, Vingron \& Argos (1989) calculate the weight of one sequence as the sum of its pairwise distances to all the other sequences, and Sibbald \& Argos (1990) weight each sequence according to its Voronoi volume in sequence space.

The basis for our weighting scheme is using the distances between the sequences to cluster them in a bifurcating tree (Fitch \& Margoliash, 1967). To construct the tree, we use the method of arithmetic averaging of pairwise distances (Nei, 1987: Sneath \&
Sokal, 1973). where the distance between se(juences is measured by percentage residue identity. This method has been implemented in the program (LLLSTALV (Higgins \& Sharp. 1988). Since no known tree-construction method consistently makes better trees than other methods. we chose the distance averaging method because of its conceptual simplicity and modest requirements for (' $\mathrm{Pl}$ ' time and memory. However, our weighting scheme could in principle be applied to any rooted tree. independent of the algorithm used to construct the tree.

We call each point of bifurcation in the tree a node. The vertical length of the edges connecting two sequences to their common ancestral node represents the average sequence identity between the two clusters of sequences, i.e. the left and right subtrees of the ancestral node. In our usage. a subtree can contain many sequences or just a single sequence. An example of a bifurcating tree is drawn in Figure 4.

To calculate weights for sequences we count

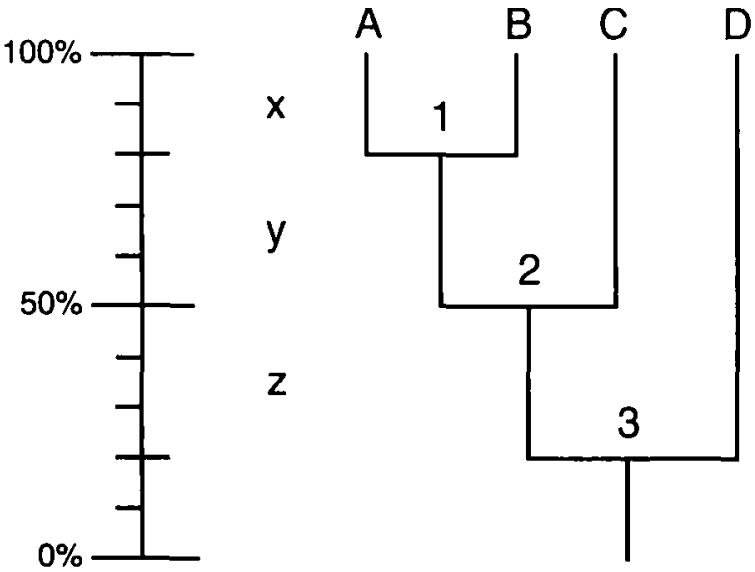

Figure 4. A worked example of our weighting method. The Figure show's a bifurcating tree with 4 sequences; $\mathrm{A}, \mathrm{B}, \mathrm{C}$ and $\mathrm{D}, \mathrm{A}$ and $\mathrm{B}$ are $80^{\circ}$ identical: the average identity between $\mathrm{C}$ and $\mathrm{A}$ or $\mathrm{B}$ is $50 \%$ : and the average identity between $\mathrm{D}$ and $\mathrm{A}$. B. or $\left({ }^{\prime}\right.$ is $20^{\circ}$. The weights for each sequence, denoted $w(s)$, are calculated by visiting the nodes sequentially (first node 1. then 2, and finally 3), and adding increments to the total weight at each node. At the end the final weights are normalized, so that the average weight is 1 . The calculation is summarized below:

\begin{tabular}{|c|c|c|c|c|}
\hline & A & B & $\mathrm{C}$ & I) \\
\hline$w(s)$ at start & 0 & 0 & 0 & 0 \\
\hline Added at 1 & $x=20$ & $x=20$ & 0 & 0 \\
\hline Added at 2 & $\frac{y}{2}=15$ & $\frac{y}{2}=15$ & $x+y=50$ & 0 \\
\hline Added at 3 & $z \frac{x+\frac{y}{2}}{3 x+2 y}=8.75$ & $2 \frac{x+\frac{y}{2}}{3 x+2 y}=8.75$ & $z \frac{x+y}{3 x+2 y}=13$ & $x+y+z=80$ \\
\hline $\begin{array}{l}w(s) \text { at end } \\
\text { normalized }\end{array}$ & $\begin{array}{l}4.3 \cdot 8 \\
0 \cdot 76\end{array}$ & $\begin{array}{l}43 \cdot 8 \\
0 \cdot 76\end{array}$ & $\begin{array}{c}63 \\
1 \cdot 09\end{array}$ & $\begin{array}{c}80 \\
1 \cdot 39\end{array}$ \\
\hline
\end{tabular}


distances between nodes in a tree. A shared edge. or distance, between subtrees will give rise to a shared weight increment for all the sequences in each subtree, according to the proportions already established within the subtree. At each node we calculate the weight increment for the sequences in the subtree above and continue doing so in a recursive fashion from the node closest to $100 \%$ identity (leaves) to the one closest to $0 \%$ (root). The relative weights of the sequences in a subtree are hence fixed when the weights for that subtree are calculated: afterwards they may change in absolute value but their relative value (to each other) will remain unchanged.

Our algorithm can be written as follows. All sequences initially have a weight of 0 . We traverse the tree by visiting each node, going from $100^{\circ}$; (leaves) to $0 \%$ (root), and for that node determine the weight increment for the left and right subtrees. The left subtree increment applies to all sequences in this subtree, and likewise for right subtrce increment. The weight added to a subtree is the length of the edge connecting it to the node currently being visited. The length of this edge is measured from the last. previously visited node of the subtree. It is apportioned between the sequences, so that at each node the sequence weights are updated according to the following formula

$$
u(s, b) \leftarrow u(s, b)+I)(b) F(s, b),
$$

where $b$ is $\mathrm{L}$, or $\mathrm{R}$ for the left or right subtree, $\therefore$ runs over all sequences in a subtree, $L(b)$ is the edge length to be apportioned. $w(s, b)$ is the current weight of sequence $s$ in subtree $t$,

and $F(s, b)$ is the weight fraction of sequence $s$ in the subtree $t$, i.e.

$$
F(s, b)= \begin{cases}1, & w(s, b)=0 \\ w(s, b), & \text { otherwise } \\ \sum_{s} u(s, b) & \end{cases}
$$

The first ('ase of the formula $(u(s, b)=0)$ is used when a node is directly connected to a sequence. In this aase. the comnecting edge is unshared, and the formula apportions its length completely to the sequence.

Figure 4 presents a completely worked out example of the calculation of weights for a simple tree.

As shown in Table 10A, on easy-to-classify sequences such as AAAAAAAAAA and BBBBBB$B B B B$, our scheme gives completely intuitive weights. Furthermore, as shown in Table $10 \mathrm{~B}$, in calculations on real sequences, it gives results that are similar to those of Sibbald \& Argos (1990). In principle (i.e. given the same assumptions we made earlier about sequence space), sibbald \& Argos' weighting scheme should give the correct results. However, it requires a Monte-Carlo integration, which is time-consuming to perform and adds an element of randomness to the calculation (i.e. two
Table 10

Comparison of our weighting scheme with other methods and with intuition

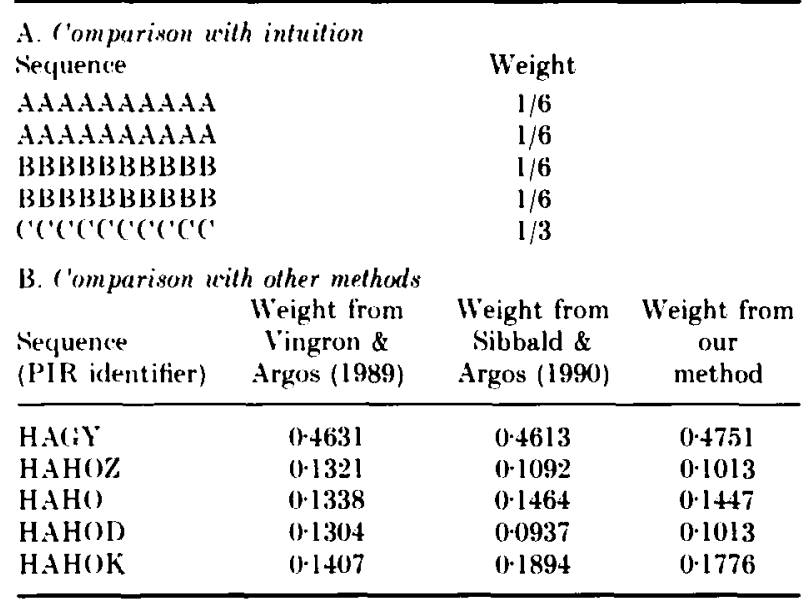

Both parts (A and B) are adapted from Sibbald \& Argos (1990). For simple sequences, the weights our method assigns to the seyuences are in good accord with intuition. For 5 globin sequences, our method produces similar weights to those of Sibbald \& Argos (1990). Note. to make the comparison with Sibbald \& Argos clearer, the weights in this Table are normalized so that the sum of the weights is 1 . This normalization follows a different convention from that used in the rest of the text.

Table 11

Effect of the weighting scheme on our results

\begin{tabular}{|c|c|c|c|}
\hline \multicolumn{4}{|l|}{$\begin{array}{l}\text { (alculated with weighting (from } \\
\text { Table 6) }\end{array}$} \\
\hline Number of sequences. $N$ & 568 & 40 & 24 \\
\hline S.I). in core volume. $\frac{\sigma}{V}(\%)$ & $2 \cdot 6$ & 1.5 & $2 \cdot 2$ \\
\hline ('alculated without weighting & & & \\
\hline S.D. in core volume $\frac{\sigma}{\Gamma}\left({ }_{0}\right)$ & $2 \cdot 2$ & $1 \cdot 4$ & $2 \cdot 0$ \\
\hline
\end{tabular}

Globins Plas.-Az. DHFR

This Table shows the effect of the weighting scheme on our principal result. the volume variation of the whole core. In all 'ases. the weighting scheme tended to increase the observed volume variation. It gave greater weight to under-represented sequences in the alignment. and these sequences tended (perhaps obviously) to differ more from the mean volume than better represented sequences. Note also that the effect of the weighting scheme was more pronounced in the larger globin alignment.

separate calculations of the weights will not give exactly the same answers). Our method, in contrast, is fast and involves no random numbers (so it gives exactly the same results each time).

As discussed in the main body of the text and shown in Table 11, our weighting method did not qualitatively affect our conclusions about volume variation in the protein core. Rather, it gave us more confidence in the quantitative accuracy of our results.

\section{References}

Adman. E. T. (1991). Structure and function of copper containing proteins. Curr. Opin. Struct. Biol. 1. 895-904.

Altschul, S. F.. Carroll R. J. \& Lipman, D. J. (1989). Weights for data related by a tree. J. Mol. Biol. 207 , $647-653$. 
Arents, G. A. \& Love. W. E. (1989). (Alycera dibranchiata hemoglobin. Structure and refinement at $1.5 \mathrm{~A}$ resolution. J. Mol. Biol. 210. 149-161.

Arutyunyan, E. G.. Kuranova. I. P., Vainshtein B. K. \& Steigemann. W. (1980). X-ray structural investigation of leghemoglobin. VI. Structure of acetateferrileghemoglobin at a resolution of $2 \cdot 0 \mathrm{~A}$. Kristallografiya ( $(S S R), 25.80$.

Bairoch. A. \& Boeckmann, B. (1992). The Swiss-Prot protein-sequence data-bank. Nurl. Arids Rps. 20. 2019-2022.

Baker. E. N. (1988). Structure of azurin from Alraligenes denitrificans. Refinement at $1.8 \AA$ and romparison of the two crystallographically independent molecules. J. Mol. Biol. 203. 1071 1095.

Barker. W. C.. George. D. (A.. Mewes H. W. \& Tsugita. A. (1992). The PIR-international protein sequence database. Nucl. Acids Res. 20, 2023-2026.

Bashford. D.. Chothia ('. \& Lesk. A. M. (1987). Determinants of a protein fold: unique features of the globin amino acid sequences. $J$. Mol. Biol. 196. 199-216.

Bernstein, F. C.. Koetzle, T. F.. Williams, ('. J. B.. Meyer Jr. E. F.. Brice, M. D.. Rodgers, J. R.. Kennard. (). Shimanouchi. T. \& Tasumi. M. (1977). The protein data bank: a computer-based archival tile for macromolecular structures. $J$. Mol. Biol. 112. 535-542.

Bolin. J. T.. Filman. D. J.. Matthews. D). A. Hamlin R. (') \& Kraut. J. (1982). ('rystal structures of Escherichin coli and Lactobacillus raspi, dihydrofolate reductase refined at $1.7 \AA$ resolution. I. (ieneral features and binding of methotrexate. J. Biol. ('hem. 257. I3650-13662.

Bolognesi. M.. Onesti. S.. Gatti. G.. Coda. A.. Ascenzi P. \& Brunori. M. (1989). Aplysia limarina myoglobin. Crystallographic analysis at $1 \cdot 6$ A resolution. $J$. . Mol. Biol. 205. 529-544.

Chothia. C. \& Lesk. A. M. (1986). The relation between the divergence of sequence and structure in proteins. EMBO J. 5. 823-826.

Chothia, C. \& Lesk. A. M. (1987). The evolution of protein structures. ('old Spring Harbor Symp. Quant. Biol. LII. 399-405.

Davies. J. F.. Delcamp, T. I.. Prendergast. $\mathrm{X}$. ,J. Ashford. A., Freisheim. H. \& Kraut. J. (1993a). Crystal structures of recombinant human dihydrofolate reductase complexed with folate and 5-deazofolate. Linpublished data bank entry.

Davies, J. F.. Matthews. D. A.. Oatley. S. J., Kaufman. B. T.. Xuong, N. H. \& Kraut. J. (1993b). Retined erystal structures of chicken liver dihydrofolate reductase. $3 \AA$ apo-enzyme and $1.7 \AA \mathrm{NAl}$ ) $\mathrm{A}$ holoenzyme complex. Unpublished data bank entry.

Eriksson. A. E., Baase. W. A.. Zhang. X. J.. Heinz. D. W.. Blaber. M.. Baldwin. E. P. \& Matthews. B. W' (1992). Response of a protein structure to cavity creating mutations and its relation to the hydrophobic effect. Science, 255. 178-183.

Felsenstein, J. (1985). Phylogenies and the comparative method. Am. Nat. 125, 1-15.

Fermi, G. \& Perut\%, M. F. (1981). Haemoglobin and Myoglobin. Clarendon Press, Oxford.

Fermi, G., Perutz, M. F.. Shaanan. B. \& Fourme. IR. (1984). The crystal structure of human deoxyhaemoglobin at $1.74 \AA$ resolution. J. Mol. Biol. 175 . 159-174.
Finkelstein. A. V. \& Ptitsyn. (). B. (1987). Why do globular proteins tit the limited set of folding patterns! Prog. Biophy.s. Mol. Biol. 50. 171 190.

Finkelstein. A. Y.. (iutun. A. I. \& Badretdinor. A. Y. (1993). Why are the same protein folds used to perform different functions! FEBSS Leflers. 325. $23-28$.

Fitch. W. M. \& Margoliash. E. (1967). ('onstruction of phylogenetic trees. siriencr. 155, 279284.

(iuss, J. M. \& Freeman. H. ('. (1983). Structure of oxidized poplar plastocyanin at $1 \cdot 6$ A resolution. J. Mol. Biol. 169. 521-562.

Higgins. I). (i. \& Sharp. P. M. (1988). ('I,LSTAL: a package for performing multiple sequence alignment on a microcomputer. Ciene. 73. 237-244.

Honzatko. R. B.. Hendrickson. W. A. \& Love. W. F (1985). Refinement of a molecular model for lamprey hemoglobin from Petromyzon marinus. J. Mol. Biol 184. $1+7-164$.

Janin. J. (1979). Surface and inside volumes in globular proteins. Nature (London). 277, 491-49:

Kendrew. J. ('. \& Watson. H. ('. (1966). Stabilizing Interactions in Gilobular Proteins. In Principles of Biomolecular Organization (Wolstenholme. (: E. W. \& $0^{\circ}$ ('onnor. M.. eds). .J. \& A. ('hurchill. London.

Lee. B. \& Richards. F. M. (1971). The interpretation of protein structures: estimation of static acesessibility. J. Mol. Biol. 55. 379-400.

Lesk. A. M. \& ('hothia. ('. (1980)). How different amino acid sequences determine similar protein structures: the structure and evolutionary dynamies of the globins. J. Mol. Biol. 136. 225-270.

Lim. V. I. \& Ptitsyn. (). B. (1970). On the constancy of the hydrophobic nucleus volume in molecules of myoglobins and hemoglobins. Mol. Biol. (ISSSR). 4. $37 \cdot 2-38 \cdot 2$.

Morris. A. L.. Macarthur. W. W., Hutchinson. E. (i. \& Thornton. J. Y. (1992). Stereochemical quality of protein-structure coordinates. Proteins: Struerl. Funrt. Gienet. 12. 345-364.

Murzin. A. (i. \& Finkelstein, A. V. (1988). (ieneral architecture of the $\alpha$-helical globule. \% Mol. Biol. 204. $749-769$.

Nei, M. (1987). Molecular Erolutionary (ienetir's. Columbia L'niversity Press. New York.

Phillips. S. E. V. \& Nchoenborn. B. P. (1981). Neutron diffraction reveals oxygen histidine hydrogen bond in oxymyoglobin. Nature (London) , 292, 81-82.

Ptitsyn. O. B. \& Volkenstein. M. V. (1986). Protein structures and the neutral theory of evolution. I. Biol. struct. Dynam. 4, 137-156.

Richards. F. M. (1974). The interpretation of protein structures: total volume. group volume distributions and packing density. J. Mol. Biol. 82. 1-14.

Sibbald. P. R. \& Argos. P. (1990). Weighting aligned protein or nucleic acid sequences to correct for unequal representation. I. Mol. Biol. 216. 813-818.

Nineath. P. H. A. \& Sokal. R. R. (1973). Numerical Traxonomy. W. H. Freeman. San Francisco.

Steigemann. W. \& Weber. E. (1981). Structure of erythrocruorin in different ligand states refined at $1.4 \AA$ resolution. J. Mol. Biol. 127. 309-388.

Vingron. M. \& Argos. P. (1989). A fast and sensitive multiple sequence alignment algorithm. $(A B I O S, 5$. $115-121$. 